\title{
Development of an MR-compatible DOI-PET detector module
}

\author{
Qingyang Wei ${ }^{1,3^{*}}$, Shi Wang ${ }^{2,3}$, Tianpeng $X u^{2,3}$, Yunpeng Gao ${ }^{2,3}$, Yaqiang Liü ${ }^{2,3}$, Tianyu Ma ${ }^{2,3}$ \\ From PSMR 2015: 4th Conference on PET/MR and SPECT/MR \\ La Biodola, Isola d'Elba, Italy. 17-21 May 2015
}

${ }^{1}$ Department of Electrical Engineering, Tsinghua University, Beijing, China
Silicon Photomultiplier (SiPM) is a promising sensor for MR-compatible PET systems. In this paper, we developed a compact 2-layer DOI-PET detector. The top layer is a $15 \tilde{A} 15$ LYSO array, and the crystal size is $2 \times 2 \times 7 \mathrm{~mm}^{3}$. The bottom layer is a $16 \tilde{A} 16$ array with the same size crystals. There is half-crystal offset between two layers in both transverse directions. The detector is coupled to an 8 Ã8 SiPM array (MicroFB-30035SMT, Sensl). Sixty-four channels of SiPMs are read out by an ASIC chip with in-chip multiplexing resistor networks in the form of two position and one energy analog signals, and are then converted to wave-form digital signals with $80 \mathrm{MHz}$ 12-bit ADC chips. The energy is calculated by averaging the 3 points around the peak of the pulse. Flood images with two $22 \mathrm{Na}$ point sources irradiated on the top and at the bottom of the detector module were acquired. The results show that the detector module achieves good crystal identification capability in both layers with an average energy resolution of $17.1 \%$ at $511 \mathrm{keV}$.

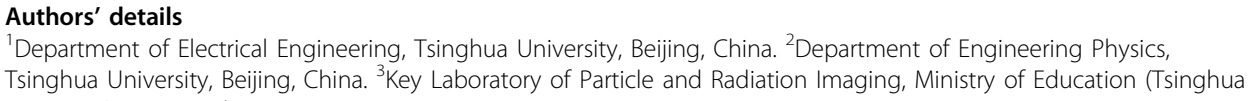

Published: 18 May 2015

doi:10.1186/2197-7364-2-S1-A4

Cite this article as: Wei et al:: Development of an MR-compatible DOI-PET detector module. EJNMMI Physics 2015 2(Suppl 1):A4.

\section{SpringerOpen ${ }^{\circ}$}

(c) 2015 Wei; licensee Springer. This is an Open Access article distributed under the terms of the Creative Commons Attribution License (http://creativecommons.org/licenses/by/4.0), which permits unrestricted use, distribution, and reproduction in any medium, provided the original work is properly cited. 